\title{
Characterisation of function spaces via mollification; fractal quantities for distributions
}

\author{
Hans Triebel \\ (Communicated by Victor Burenkov)
}

2000 Mathematics Subject Classification. 46E35, 42B35, 28A80, 42C40.

Keywords and phrases. Besov spaces, fractal quantities, multifractal quantities.

\footnotetext{
Abstract. The aim of this paper is twofold. First we characterise elements $f$ belonging to the Besov spaces $B_{p q}^{s}\left(\mathbb{R}^{n}\right)$ with $s \in \mathbb{R}, 0<p \leq \infty, 0<q \leq \infty$, in terms of their mollifications. Secondly we use these results to study multifractal quantities for distributions generalising well-known corresponding quantities for Radon measures.
}

\section{Introduction}

First we describe our motivation for this piece of work. Let $\mu$ be a positive Radon measure in $\mathbb{R}^{n}$ with

$$
\Gamma=\operatorname{supp} \mu \subset\{x:|x|<1\}, \quad 0<\mu\left(\mathbb{R}^{n}\right)<\infty .
$$

Let $Q_{j m}$ be cubes in $\mathbb{R}^{n}$, centred at $2^{-j} m$ with side-length $2^{-j+1}$ where $j \in \mathbb{N}_{0}$ and $m \in \mathbb{Z}^{n}$. Let

$$
\mu_{p q}^{\lambda}=\left(\sum_{j=0}^{\infty} 2^{j \lambda q}\left(\sum_{m \in \mathbb{Z}^{n}} \mu\left(Q_{j m}\right)^{p}\right)^{\frac{q}{p}}\right)^{\frac{1}{q}}
$$


where $0<p \leq \infty, 0<q \leq \infty$, and $\lambda \in \mathbb{R}$ (with the usual modification if $p$ and/or $q$ are infinite). Since $\mu$ is Radon there is an one-to-one relation between $\mu$ and the tempered distribution $T_{\mu} \in S^{\prime}\left(\mathbb{R}^{n}\right)$ generated by $\mu$,

$$
T_{\mu}: \quad \varphi \mapsto \int_{\mathbb{R}^{n}} \varphi(x) \mu(d x), \quad \varphi \in S\left(\mathbb{R}^{n}\right) .
$$

This justifies (with the usual abuse of notation) our writing $\mu \in S^{\prime}\left(\mathbb{R}^{n}\right)$ in the sequel. Let $B_{p q}^{s}\left(\mathbb{R}^{n}\right)$ be the usual Besov spaces. We describe some results obtained in [18] which are of relevance for our later purposes and which are the starting point of this paper. Let

$$
0 \leq t=\frac{1}{p}<\infty, \quad 0<q \leq \infty, \quad \text { and } \quad s<0 .
$$

Then

(1.4) $\mu \in B_{p q}^{s}\left(\mathbb{R}^{n}\right)$ if, and only if, $\mu_{p q}^{\lambda}<\infty$ with $s=\lambda+n(t-1)$ and, in addition,

$$
\left\|\mu \mid B_{p q}^{s}\left(\mathbb{R}^{n}\right)\right\| \sim \mu_{p q}^{\lambda}
$$

where the equivalence constants are independent of $\mu$ with (1.1). Let now $\mu$ be, in addition, singular, which means

$$
\Gamma=\operatorname{supp} \mu \subset\{x:|x|<1\}, \quad 0<\mu\left(\mathbb{R}^{n}\right)<\infty, \quad|\Gamma|=0,
$$

where $|\Gamma|$ is the Lebesgue measure of $\Gamma$. Let for $\mu$ with (1.6) and $0 \leq t=$ $\frac{1}{p}<\infty$,

$$
\lambda_{\mu}(t)=\sup \left\{\lambda: \mu_{p \infty}^{\lambda}<\infty\right\}
$$

and

$$
s_{\mu}(t)=\sup \left\{s: \mu \in B_{p \infty}^{s}\left(\mathbb{R}^{n}\right)\right\} .
$$

For $\mu$ with (1.6) and $t \geq 0$ both $\lambda_{\mu}(t)$ and $s_{\mu}(t)$ are concave functions where $\lambda_{\mu}(1)=s_{\mu}(1)=0$. Furthermore, $s_{\mu}(t)$ is monotonically increasing (which means not decreasing),

$$
n(t-1) \leq s_{\mu}(t)=\lambda_{\mu}(t)+n(t-1) \leq 0 \quad \text { if } \quad 0 \leq t \leq 1,
$$

and

$$
0 \leq \lambda_{\mu}(t)+n(t-1) \leq s_{\mu}(t) \leq n(t-1) \text { if } t>1,
$$

Figure 1. We refer for details to [18]. The characterisation (1.4) with (1.3), and then also (1.5), is valid for all Radon measures $\mu$ according to (1.1). But there are no characterisations of this type if $s>0$ and it is one aim of this paper to find suitable substitutes (for measures and, more general, for distributions). On the other hand we discussed in [18] the problem of whether the equality in the middle of (1.9) for $0 \leq t \leq 1$ can be extended 
to $t>1$. There are a few affirmative examples such as the restriction of the Hausdorff measure $\mathcal{H}^{d}$ to $d$-sets, but no general assertion (and no counterexample). It is the second aim of this paper to contribute to this problem (for measures and, more general, for distributions) after a suitable extension of (1.4), (1.5) to all $0<p \leq \infty, 0<q \leq \infty$ and $s \in \mathbb{R}$ has been found. The interest in quantities of type $\lambda_{\mu}(t)$, which can also be written as

$$
\lambda_{\mu}(t)=-t \limsup _{j \rightarrow \infty} \frac{1}{j} \log \left[\sum_{m \in \mathbb{Z}^{n}} \mu\left(Q_{j m}\right)^{\frac{1}{t}}\right] \quad \text { if } \quad 0<t<\infty,
$$

and

$$
\lambda_{\mu}(0)=-\limsup _{j \rightarrow \infty} \frac{1}{j} \log \left[\sup _{m \in \mathbb{Z}^{n}} \mu\left(Q_{j m}\right)\right],
$$

(log taken to base 2), comes from the multifractal formalism for measures. We refer to [5], Ch. 11; [10]; [6] and the literature given there (a few further relevant papers may also be found in [18]). In other words, it is one motivation of this paper to ask for a suitable substitute of $\lambda_{\mu}(t)$ such that we have afterwards the same equality in (1.10) as in the middle of (1.9) when penetrating from $0 \leq t \leq 1$ into the somewhat

$$
\text { gloomy region }\{(t, s): t>1,0 \leq s \leq n(t-1)\}
$$

of the otherwise lucid

$$
\text { function spaces universe }\{(t, s): t \geq 0, s \in \mathbb{R}\},
$$

Next we describe the main intentions of this paper. Our first aim to extend (1.3) -(1.5) to the whole function space universe according to (1.13) is not restricted to measures but applies to any $f \in B_{p q}^{s}\left(\mathbb{R}^{n}\right)$. There is a counterpart for the spaces $F_{p q}^{s}\left(\mathbb{R}^{n}\right)$, but this will not be considered in this paper. For this purpose one needs the mollifications

$$
K(\tau, f)(x)=\int_{\mathbb{R}^{n}} K(y) f(x-\tau y) d y=\tau^{-n} \int_{\mathbb{R}^{n}} K\left(\frac{x-y}{\tau}\right) f(y) d y
$$

of $f \in S^{\prime}\left(\mathbb{R}^{n}\right)$ (with the usual interpretation) for $\tau>0$ where the kernel $K$ is a real non-negative $C^{\infty}$ function in $\mathbb{R}^{n}$ with, say,

$$
K(y)>0 \Longleftrightarrow|y|<c \text { and } \widehat{K}(0)=1
$$

for some (suitable) $c>0$. Recall that for $f \in S^{\prime}\left(\mathbb{R}^{n}\right)$,

$$
K(\tau, f) \rightarrow f \quad \text { in } \quad S^{\prime}\left(\mathbb{R}^{n}\right) \quad \text { if } \quad \tau \rightarrow 0 .
$$




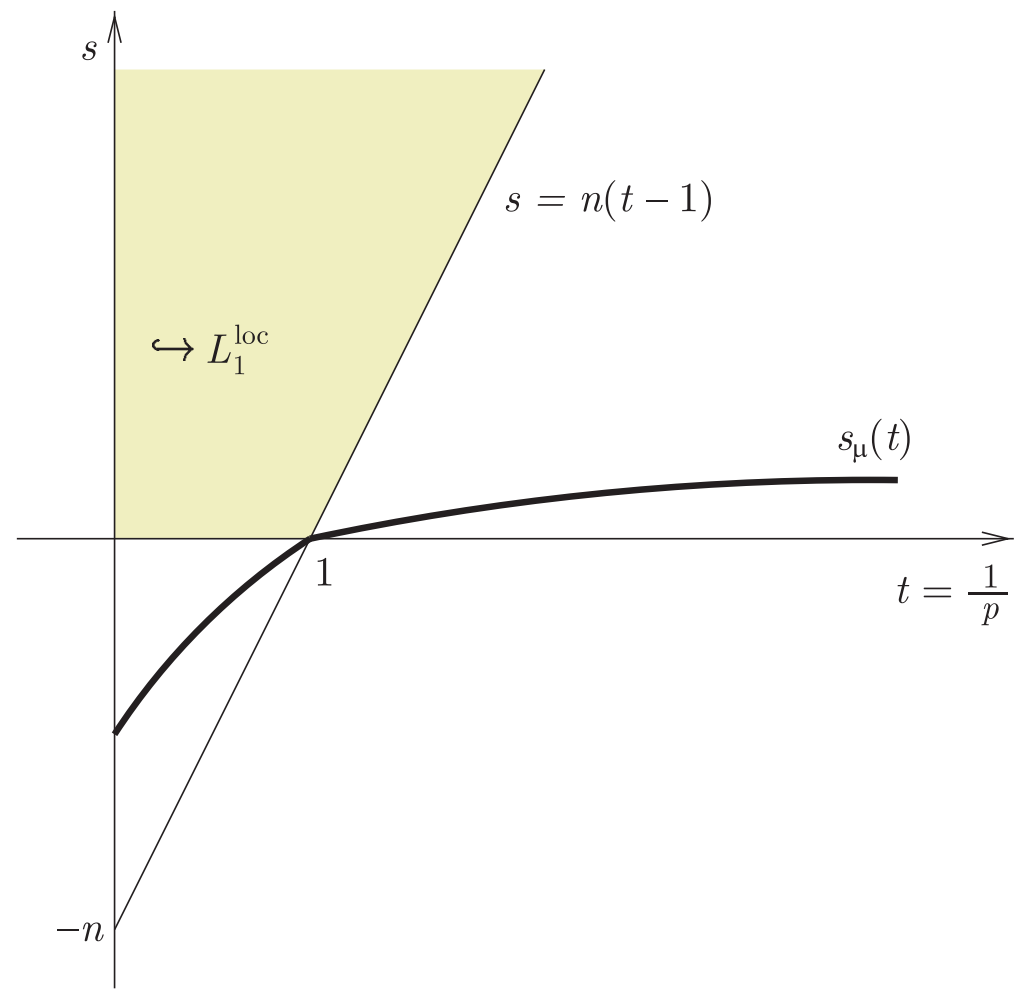

Figure 1

Now (1.2) can be reformulated by

$$
\mu_{p q}^{\lambda} \sim\left(\sum_{j=0}^{\infty} 2^{j q(\lambda-n)}\left(\sum_{m \in \mathbb{Z}^{n}} K\left(2^{-j}, \mu\right)^{p}\left(2^{-j} m\right)\right)^{\frac{q}{p}}\right)^{\frac{1}{q}} .
$$

In this version one can extend (1.3) - (1.5) first to all $f \in B_{p q}^{s}\left(\mathbb{R}^{n}\right)$ and secondly to the whole function space universe according to (1.13). For this purpose one has to replace the kernel in the mollifications $K\left(2^{-j}, \mu\right)(x)$ by suitable linear combinations

$$
\begin{aligned}
K^{M}\left(2^{-j}, f\right)(x) & =\sum_{m \in \mathbb{Z}_{M}^{n}} d_{m}^{M} K\left(2^{-j}, f\right)\left(x+2^{-j} m\right) \\
& =2^{j n} \int_{\mathbb{R}^{n}}\left(\mathbb{D}^{M} K\right)\left(2^{j}(x-y)\right) f(y) d y,
\end{aligned}
$$


where

$$
\mathbb{Z}_{M}^{n}=\left\{m \in \mathbb{Z}^{n}: \sum_{k=1}^{n}\left|m_{k}\right| \leq M\right\}, \quad M \in \mathbb{N}_{0},
$$

are rhombohedrons, $d_{m}^{M} \in \mathbb{Z}$; and $\mathbb{D}^{M}$ is the $M$ th iteration of the symmetric difference

$$
(\mathbb{D} g)(x)=\frac{1}{4} \sum_{k=1}^{n}\left(g\left(x+e_{k}\right)-2 g(x)+g\left(x-e_{k}\right)\right), \quad x \in \mathbb{R}^{n},
$$

with the unit vectors $e_{k}$ pointing in the $k$ th direction of the axes of coordinates. In other words, for given $s \in \mathbb{R}, 0<p \leq \infty$, and $0<q \leq \infty$ we characterise those elements $f \in S^{\prime}\left(\mathbb{R}^{n}\right)$ which belong to $B_{p q}^{s}\left(\mathbb{R}^{n}\right)$ in terms of their mollifications

$$
\left\{K\left(2^{-j}, f\right)(x): j \in \mathbb{N}_{0}, x \in \mathbb{R}^{n}\right\},
$$

including linear combinations and some maximal functions. Afterwards we apply the outcome not only to Radon measures $\mu$ with (1.6) modifying the multifractal quantities (1.7); hence (1.11), (1.12), and (1.9), (1.10); but also to compactly supported distributions $f \in S^{\prime}\left(\mathbb{R}^{n}\right)$.

The plan of the paper is the following. In Section 2 we collect some definitions, notation and prerequisites. In particular we recall in Theorem 1 a characterisation of $B_{p q}^{s}\left(\mathbb{R}^{n}\right)$ in terms of maximal functions following V.S. Rychkov, [13]. In Section 3 we describe the characterisation of the spaces $B_{p q}^{s}\left(\mathbb{R}^{n}\right)$ by means of mollifications $K^{M}$ according to (1.15), Theorem 2. In Section 4 we discuss some consequences of these results with respect to fractal quantities for distributions and for measures $\mu$ according to (1.6), Theorem 3.

\section{Preliminaries and basic assertions}

2.1. Basic notation. We use standard notation. Let $\mathbb{N}$ be the collection of all natural numbers and $\mathbb{N}_{0}=\mathbb{N} \cup\{0\}$. Let $\mathbb{R}^{n}$ be euclidean $n$ space, where $n \in \mathbb{N}$. Put $\mathbb{R}=\mathbb{R}^{1}$. As usual, $\mathbb{Z}$ is the collection of all integers. Furthermore, $\mathbb{Z}^{n}$, where $n \in \mathbb{N}$, denotes the lattice of all points $m=\left(m_{1}, \ldots, m_{n}\right) \in \mathbb{R}^{n}$ with $m_{j} \in \mathbb{Z}$. Let $S\left(\mathbb{R}^{n}\right)$ be the Schwartz space of all complex-valued rapidly decreasing, infinitely differentiable functions in $\mathbb{R}^{n}$. By $S^{\prime}\left(\mathbb{R}^{n}\right)$ we denote its topological dual, the space of tempered distributions in $\mathbb{R}^{n}$. Furthermore, $L_{p}\left(\mathbb{R}^{n}\right)$ with $0<p \leq \infty$, is the standard quasi-Banach space of all complex-valued functions with respect to the Lebesgue measure, quasi-normed by

$$
\left\|f \mid L_{p}\left(\mathbb{R}^{n}\right)\right\|=\left(\int_{\mathbb{R}^{n}}|f(x)|^{p} d x\right)^{\frac{1}{p}}
$$


with the usual modification if $p=\infty$.

Let $\mu$ be a Radon measure in $\mathbb{R}^{n}$ with

$$
\Gamma=\operatorname{supp} \mu \subset\{x:|x|<1\} \quad \text { and } \quad 0<\mu\left(\mathbb{R}^{n}\right)<\infty,
$$

and let $T_{\mu}$,

$$
T_{\mu}: \quad \varphi \mapsto \int_{\mathbb{R}^{n}} \varphi(x) \mu(d x), \quad \varphi \in S\left(\mathbb{R}^{n}\right),
$$

be the tempered distribution generated by $\mu$. Since $S\left(\mathbb{R}^{n}\right)$ is dense in the Banach space $C_{0}\left(\mathbb{R}^{n}\right)$ of all bounded continuous functions in $\mathbb{R}^{n}$ tending to zero if $|x| \rightarrow \infty$, one can interprete $T_{\mu}$ as a linear and continuous functional on $C_{0}\left(\mathbb{R}^{n}\right)$. Then it follows by the Riesz representation theorem, [9], Theorem 6.6, p. 97, that there is an one-to-one relation between the above Radon measure $\mu$ and the generated distribution $T_{\mu}$. This justifies to write $\mu \in S^{\prime}\left(\mathbb{R}^{n}\right)$ (with the usual slight abuse of notation).

If $\varphi \in S\left(\mathbb{R}^{n}\right)$ then

$$
\widehat{\varphi}(\xi)=(F \varphi)(\xi)=(2 \pi)^{-\frac{n}{2}} \int_{\mathbb{R}^{n}} e^{-i x \xi} \varphi(x) d x, \quad \xi \in \mathbb{R}^{n},
$$

denotes the Fourier transform of $\varphi$. As usual, $F^{-1} \varphi$ or $\varphi^{\vee}$ stands for the inverse Fourier transform, given by the right-hand side of (2.1) with $i$ in place of $-i$. Here $x \xi$ denotes the scalar product in $\mathbb{R}^{n}$. Both $F$ and $F^{-1}$ are extended to $S^{\prime}\left(\mathbb{R}^{n}\right)$ in the standard way.

2.2. Function spaces. Let $\varphi \in S\left(\mathbb{R}^{n}\right)$ with

$$
\varphi(x)=1 \text { if }|x| \leq 1 \quad \text { and } \quad \varphi(y)=0 \text { if }|y| \geq \frac{3}{2} .
$$

We put $\varphi_{0}=\varphi, \varphi_{1}(x)=\varphi\left(\frac{x}{2}\right)-\varphi(x)$, and

$$
\varphi_{k}(x)=\varphi_{1}\left(2^{-k+1} x\right), \quad x \in \mathbb{R}^{n}, \quad k \in \mathbb{N} .
$$

Then, since

$$
1=\sum_{k=0}^{\infty} \varphi_{k}(x) \text { for all } x \in \mathbb{R}^{n},
$$

the $\varphi_{k}$ form a dyadic resolution of unity. Recall that $\left(\varphi_{k} \widehat{f}\right)^{\vee}$ is an entire analytic function on $\mathbb{R}^{n}$ for any $f \in S^{\prime}\left(\mathbb{R}^{n}\right)$. In particular, $\left(\varphi_{k} \widehat{f}\right)^{\vee}(x)$ makes sense pointwise. 
Definition 1. Let $s \in \mathbb{R}, 0<p \leq \infty$, and $0<q \leq \infty$. Then $B_{p q}^{s}\left(\mathbb{R}^{n}\right)$ is the collection of all $f \in S^{\prime}\left(\mathbb{R}^{n}\right)$ such that

$$
\left\|f \mid B_{p q}^{s}\left(\mathbb{R}^{n}\right)\right\|=\left(\sum_{j=0}^{\infty} 2^{j s q}\left\|\left(\varphi_{j} \widehat{f}\right)^{\vee} \mid L_{p}\left(\mathbb{R}^{n}\right)\right\|^{q}\right)^{\frac{1}{q}}
$$

(with the usual modification if $q=\infty$ ) is finite.

Remark 1. There is a second scale of spaces of this type, denoted by $F_{p q}^{s}\left(\mathbb{R}^{n}\right.$ ) (now with $p<\infty$ ) where one has to replace the $\ell_{q}\left(L_{p}\right)$-quasinorm in (2.3) by the $L_{p}\left(\ell_{q}\right)$-quasi-norm (changing the order of summation and integration). The main assertions of this paper for $B_{p q}^{s}\left(\mathbb{R}^{n}\right)$ have a counterpart for the spaces $F_{p q}^{s}\left(\mathbb{R}^{n}\right)$. But we restrict ourselves here to the spaces $B_{p q}^{s}\left(\mathbb{R}^{n}\right)$. They are simpler to handle and they are coming in naturally in the context of Radon measures as described in the Introduction. The theory of these spaces in its full extent may be found in [14] and [15]. As for more recent topics we refer to [1], [4], [12], [16], [17]. In particular, $B_{p q}^{s}\left(\mathbb{R}^{n}\right)$ (and also $F_{p q}^{s}\left(\mathbb{R}^{n}\right)$ ) are quasi-Banach spaces which are independent of the function $\varphi$ according to (2.2). This justifies our omission of the subscript $\varphi$ in the quasi-norm (2.3). Otherwise we assume that the reader is familiar with the basic assertions of these spaces. Here we only mention that the (Hilbert-) Sobolev spaces

$$
H^{s}\left(\mathbb{R}^{n}\right)=H_{2}^{s}\left(\mathbb{R}^{n}\right)=B_{2,2}^{s}\left(\mathbb{R}^{n}\right), \quad s \in \mathbb{R},
$$

with the classical Sobolev spaces

$$
W_{2}^{m}\left(\mathbb{R}^{n}\right)=H^{m}\left(\mathbb{R}^{n}\right), \quad m \in \mathbb{N}_{0},
$$

as a sub-class, and the Hölder-Zygmund spaces

$$
\mathcal{C}^{s}\left(\mathbb{R}^{n}\right)=B_{\infty \infty}^{s}\left(\mathbb{R}^{n}\right), \quad s \in \mathbb{R},
$$

are distinguished special cases. If $s>0,1<p<\infty, 1 \leq q \leq \infty$, then $B_{p q}^{s}\left(\mathbb{R}^{n}\right)$ are the well-known classical Besov spaces. The rich history of these spaces may be found in [15], Chapter 1 .

2.3. Characterisations in terms of maximal functions. Our later considerations will be based on some characterisations of the spaces $B_{p q}^{s}\left(\mathbb{R}^{n}\right)$ as introduced in Definition 1 in terms of maximal functions. Let $\psi_{0} \in S\left(\mathbb{R}^{n}\right)$, $\psi \in S\left(\mathbb{R}^{n}\right)$ and

$$
\psi_{j}(x)=\psi\left(2^{-j} x\right), \quad x \in \mathbb{R}^{n}, \quad j \in \mathbb{N} .
$$

Then, for $f \in S^{\prime}\left(\mathbb{R}^{n}\right)$,

$$
\psi_{0}(D) f(x)=\left(\psi_{0} \widehat{f}\right)^{\vee}(x)=c \int_{\mathbb{R}^{n}} \psi_{0}^{\vee}(x-y) f(y) d y
$$


and

$$
\psi_{j}(D) f(x)=\left(\psi\left(2^{-j} \cdot\right) \hat{f}\right)^{\vee}(x)=c 2^{j n} \int_{\mathbb{R}^{n}} \psi^{\vee}\left(2^{j}(x-y)\right) f(y) d y, \quad j \in \mathbb{N},
$$

where $c>0$ is an unimportant constant originating from the normalisation in (2.1). Of course, (2.4), (2.5), must be interpreted as usual in terms of the $S\left(\mathbb{R}^{n}\right)$ - $S^{\prime}\left(\mathbb{R}^{n}\right)$-duality. They are $C^{\infty}$ functions in $\mathbb{R}^{n}$ of at most polynomial growth. Let for $a>0$ and $k \in \mathbb{N}_{0}$,

$$
\left(\psi_{k}^{*} f\right)_{a}(x)=\sup _{y \in \mathbb{R}^{n}} \frac{\left|\psi_{k}(D) f(x-y)\right|}{1+\left|2^{k} y\right|^{a}}, \quad x \in \mathbb{R}^{n},
$$

be the maximal functions as introduced in [11] and considered in [15], 2.3.2, p. 93, where one finds also further comments.

Theorem 1. Let $0<p \leq \infty, 0<q \leq \infty$, and $s \in \mathbb{R}$. Let $a>\frac{n}{p}$ and $s<N \in \mathbb{N}_{0}$. Let $\psi_{0} \in S\left(\mathbb{R}^{n}\right)$ and $\psi \in S\left(\mathbb{R}^{n}\right)$ satisfying the Tauberian conditions

$$
\left|\psi_{0}(x)\right|>0 \text { if }|x| \leq \varepsilon \quad \text { and } \quad|\psi(y)|>0 \text { if } \frac{\varepsilon}{2} \leq|y| \leq 2 \varepsilon
$$

for some $\varepsilon>0$ and

$$
\left(D^{\alpha} \widehat{\psi}\right)(0)=0 \quad \text { for all }|\alpha|<N .
$$

Let

$$
\left\|f \mid B_{p q}^{s}\left(\mathbb{R}^{n}\right)\right\|_{a}=\left(\sum_{k=0}^{\infty} 2^{k s q}\left\|\left(\psi_{k}^{*} f\right)_{a} \mid L_{p}\left(\mathbb{R}^{n}\right)\right\|^{q}\right)^{\frac{1}{q}}
$$

(with the usual modification if $q=\infty$ ). Then

$$
B_{p q}^{s}\left(\mathbb{R}^{n}\right)=\left\{f \in S^{\prime}\left(\mathbb{R}^{n}\right):\left\|f \mid B_{p q}^{s}\left(\mathbb{R}^{n}\right)\right\|_{a}<\infty\right\}
$$

and (2.8) is an equivalent quasi-norm in $B_{p q}^{s}\left(\mathbb{R}^{n}\right)$.

Remark 2. If $N=0$ then (2.7) is empty and there are no conditions. The above formulation coincides essentially with the theorem in [13] for the spaces $B_{p q}^{s}\left(\mathbb{R}^{n}\right)$. There is a corresponding assertion for the spaces $F_{p q}^{s}\left(\mathbb{R}^{n}\right)$ which are not considered in this paper. The theorem itself has some history. Applying [15], Corollaries 1 and 2 on p. 134, it follows that (2.8) is an equivalent quasi-norm. But we needed larger values of $N$ in (2.7) (even depending on $p$ if $p<1$ ). The next step has been done in [2], [3], arriving essentially at the above theorem, an $F_{p q}^{s}$-counterpart and even some weighted generalisations. Based on these papers, V.S. Rychkov simplified in [13] these arguments and corrected an error. The interesting case $s<0$ and $N=0$ has its own little history which we tried to collect in [17], p. 125, and which will not be repeated here. In this case one may choose $\psi=\psi_{0}$. For 
our later purposes it is convenient to replace the maximal function $\left(\psi_{k}^{*} f\right)_{a}$ in (2.6) by

$$
\left(\psi_{k}^{+} f\right)(x)=\sup _{|y| \leq 2^{-k}}\left|\psi_{k}(D) f(x-y)\right|, \quad x \in \mathbb{R}^{n}, \quad k \in \mathbb{N}_{0} .
$$

Then by the above theorem,

$$
\left|\psi_{k}(D) f(x)\right| \leq\left(\psi_{k}^{+} f\right)(x) \leq 2\left(\psi_{k}^{*}\right)_{a}(x), \quad x \in \mathbb{R}^{n},
$$

and again by [13] one can replace $\left(\psi_{k}^{*} f\right)_{a}$ in (2.8) either by $\psi_{k}(D) f$ or by $\psi_{k}^{+} f$ and one gets respective characterisations (2.9). In particular, one obtains the following characterisation.

Corollary 1. Let $p, q, s$ and also $N$ be the same numbers and $\psi_{0}, \psi$ be the same functions as in the above theorem. Let

$$
\left\|f \mid B_{p q}^{s}\left(\mathbb{R}^{n}\right)\right\|_{+}=\left(\sum_{k=0}^{\infty} 2^{k\left(s-\frac{n}{p}\right) q}\left(\sum_{m \in \mathbb{Z}^{n}}\left(\psi_{k}^{+} f\right)^{p}\left(2^{-k} m\right)\right)^{\frac{q}{p}}\right)^{\frac{1}{q}}
$$

(with the usual modification if $p$ and/or $q$ is infinite). Then

$$
B_{p q}^{s}\left(\mathbb{R}^{n}\right)=\left\{f \in S^{\prime}\left(\mathbb{R}^{n}\right):\left\|f \mid B_{p q}^{s}\left(\mathbb{R}^{n}\right)\right\|_{+}<\infty\right\} .
$$

Remark 3. This follows immediately from the above considerations, covering $\mathbb{R}^{n}$ with cubes centred at $2^{-k} m$ and with side-lengths $c 2^{-k}$ in connection with the $L_{p}$-integration of $\psi_{k}^{+} f$.

\section{Characterisations via mollification}

3.1. Some preparations. We specify $\psi_{0}$ and $\psi$ in 2.3 and, consequently, in Theorem 1 and in Corollary 1 . Let $K$ be a real non-negative $C^{\infty}$ function in $\mathbb{R}^{n}$ with

$$
K(x)>0 \text { if }|x|<c ; \quad K(y)=0 \text { if }|y| \geq c ; \quad \text { and } \quad \widehat{K}(0)=1,
$$

for some $c>0$. Let $e_{k}=(0, \ldots, 1,0 \ldots, 0)$ with 1 in the $k$ th position be the unit vector pointing in the $k$ th direction of the axes of coordinates; $k=1, \ldots, n$. Let

$$
\left(\mathbb{D}^{1} g\right)(x)=\frac{1}{4} \sum_{k=1}^{n}\left(g\left(x+e_{k}\right)-2 g(x)+g\left(x-e_{k}\right)\right), \quad x \in \mathbb{R}^{n},
$$

be the sum of the second symmetric differences and, by iteration,

$$
\mathbb{D}^{M+1} g=\mathbb{D}^{1} \mathbb{D}^{M} g, \quad M \in \mathbb{N} .
$$

Lemma 1. Let $K$ be the above function and let $M \in \mathbb{N}$. Then

$$
\left(\mathbb{D}^{M} K\right)^{\wedge}(\xi)=(-1)^{M} \widehat{K}(\xi)\left(\sum_{k=1}^{n} \sin ^{2} \frac{\xi_{k}}{2}\right)^{M}, \quad \xi \in \mathbb{R}^{n} .
$$


Proof. Let $M=1$. Then

$$
\begin{aligned}
\left(\mathbb{D}^{1} K\right)^{\wedge}(\xi) & =\frac{1}{4} \sum_{k=1}^{n}\left(e^{i \xi_{k}}-2+e^{-i \xi_{k}}\right) \widehat{K}(\xi) \\
& =\frac{1}{4} \sum_{k=1}^{n}\left(e^{i \frac{\xi_{k}}{2}}-e^{-i \frac{\xi_{k}}{2}}\right)^{2} \widehat{K}(\xi) \\
& =-\left(\sum_{k=1}^{n} \sin ^{2} \frac{\xi_{k}}{2}\right) \widehat{K}(\xi),
\end{aligned}
$$

and (3.3) follows by iteration.

Remark 4. The structure of $\mathbb{D}^{M}$ might be of some interest. By (3.3), $\mathbb{D}^{M}$ discretises $(-\Delta)^{M}$, the $M$ th power of the Laplacian $-\Delta$. Let $M \in \mathbb{N}_{0}$ and let $\mathbb{D}^{0} K=K$. Then

$$
\mathbb{Z}_{M}^{n}=\left\{m \in \mathbb{Z}^{n}: \sum_{k=1}^{n}\left|m_{k}\right| \leq M\right\}
$$

is a rhombohedron in $\mathbb{Z}^{n}$ and we have

$$
\left(\mathbb{D}^{M} g\right)(x)=2^{-2 M} \sum_{m \in \mathbb{Z}_{M}^{n}} d_{m}^{M} g(x+m), \quad x \in \mathbb{R}^{n}, \quad M \in \mathbb{N}_{0},
$$

where the integers $d_{m}^{M} \in \mathbb{Z}$ can be calculated by iteration. We give an example. Let $n=2$. Then, by induction,

$$
\operatorname{card} \mathbb{Z}_{M}^{2}=1+2 M(M+1), \quad M \in \mathbb{N}_{0} .
$$

Furthermore, $d_{(0,0)}^{0}=1$ for $M=0$;

$$
d_{(0,0)}^{1}=-4, \quad d_{(1,0)}^{1}=d_{(-1,0)}^{1}=d_{(0,1)}^{1}=d_{(0,-1)}^{1}=1,
$$

for $M=1$; and

$$
\begin{aligned}
& d_{(0,0)}^{2}=20, \quad d_{(1,0)}^{2}=d_{(-1,0)}^{2}=d_{(0,1)}^{2}=d_{(0,-1)}^{2}=-8, \\
& d_{(1,1)}^{2}=d_{(1,-1)}^{2}=d_{(-1,1)}^{2}=d_{(-1,-1)}^{2}=2, \\
& d_{(2,0)}^{2}=d_{(-2,0)}^{2}=d_{(0,2)}^{2}=d_{(0,-2)}^{2}=1,
\end{aligned}
$$

for $M=2$, etc.

3.2. Characterisations. Let $K$ be the function according to (3.1) and let

$$
K(\tau, f)(x)=\int_{\mathbb{R}^{n}} K(y) f(x-\tau y) d y=\tau^{-n} \int_{\mathbb{R}^{n}} K\left(\frac{x-y}{\tau}\right) f(y) d y
$$


be the mollifications of $f \in S^{\prime}\left(\mathbb{R}^{n}\right)$, where $\tau>0$. They are $C^{\infty}$ functions of at most polynomial growth. Furthermore, for any $f \in S^{\prime}\left(\mathbb{R}^{n}\right)$,

$$
K(\tau, f) \rightarrow f \quad \text { in } \quad S^{\prime}\left(\mathbb{R}^{n}\right) \quad \text { if } \quad \tau \rightarrow 0 .
$$

According to (3.5) we have for $\tau>0$,

$$
\begin{aligned}
K^{M}(\tau, f)(x) & =\int_{\mathbb{R}^{n}}\left(\mathbb{D}^{M} K\right)(y) f(x-\tau y) d y \\
& =2^{-2 M} \sum_{m \in \mathbb{Z}_{M}^{n}} d_{m}^{M} \int_{\mathbb{R}^{n}} K(y+m) f(x-\tau y) d y \\
& =2^{-2 M} \sum_{m \in \mathbb{Z}_{M}^{n}} d_{m}^{M} K(\tau, f)(x+\tau m) .
\end{aligned}
$$

We adapt the formulation of the following assertions to the situation as described in the Introduction with respect to Radon measures. Let, as there, $Q_{j m}$ be cubes in $\mathbb{R}^{n}$, centred at $2^{-j} m$ with side-length $2^{-j+1}$ where $j \in \mathbb{N}_{0}$ and $m \in \mathbb{Z}^{n}$. Let $M \in \mathbb{N}_{0}$ and $f \in S^{\prime}\left(\mathbb{R}^{n}\right)$. Then, for $x \in \mathbb{R}^{n}$,

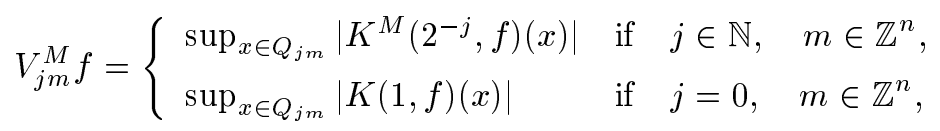

where $K^{M}$ and $K$ are given by (3.7), (3.6). If $M=0$ then $K^{M}=K$.

Theorem 2. Let $0<p \leq \infty, 0<q \leq \infty$, and $s \in \mathbb{R}$. Let $M \in \mathbb{N}_{0}$ and $2 M>s$. Let

$$
\left\|f \mid B_{p q}^{s}\left(\mathbb{R}^{n}\right)\right\|^{M}=\left(\sum_{j=0}^{\infty} 2^{j\left(s-\frac{n}{p}\right) q}\left(\sum_{m \in \mathbb{Z}^{n}}\left(V_{j m}^{M} f\right)^{p}\right)^{\frac{q}{p}}\right)^{\frac{1}{q}}
$$

(with the usual modification if $p$ and/or $q$ is infinite). Then

$$
B_{p q}^{s}\left(\mathbb{R}^{n}\right)=\left\{f \in S^{\prime}\left(\mathbb{R}^{n}\right):\left\|f \mid B_{p q}^{s}\left(\mathbb{R}^{n}\right)\right\|^{M}<\infty\right\}
$$

and (3.9) is an equivalent quasi-norm in $B_{p q}^{s}\left(\mathbb{R}^{n}\right)$.

Proof. Let

$$
\psi_{0}=\widehat{K} \quad \text { and } \quad \psi=\left(\mathbb{D}^{M} K\right)^{\wedge} .
$$

Then $K(1, f)$ coincides with $(2.4)$ and we have a corresponding assertion for $K^{M}\left(2^{-j}, f\right)$ and $(2.5)$. Furthermore, $V_{j m}^{M} f$ is an immaterial modification of (2.10) with $x=2^{-j} m$. By (3.3) we have (2.7) with $N=2 M$. Now the above theorem follows from Corollary 1 .

Remark 5. Let $s<0$. Then we may choose $M=0$ in the above theorem and in (3.8). If, in addition, $\mu$ is a Radon measure according to (1.1), then 
we have by (3.9) with $M=0$ that

$$
\left\|\mu \mid B_{p q}^{s}\left(\mathbb{R}^{n}\right)\right\|^{0} \sim\left(\sum_{j=0}^{\infty} 2^{j\left(s+n-\frac{n}{p}\right) q}\left(\sum_{m \in \mathbb{Z}^{n}} \mu\left(Q_{j m}\right)^{p}\right)^{\frac{q}{p}}\right)^{\frac{1}{q}} .
$$

This coincides with (1.3) - (1.5). If $s \geq 0$, then one has to choose $2 M>s$ and some variations (or oscillations) $V_{j m}^{M} \mu$ of the mollifications $K\left(2^{-j}, \mu\right)$ according to (3.7) come in. Hence the above theorem extends (1.3) - (1.5) from $s<0$ to $s \in \mathbb{R}$ and applies now to all $f \in B_{p q}^{s}\left(\mathbb{R}^{n}\right)$. The choice of the special kernels $\mathbb{D}^{M} K=\widehat{\psi}$ in (3.7) is convenient for the later applications of Theorem 2 to fractal quantities. But Theorem 2 itself remains valid as long as the respective kernels satisfy the hypotheses of Theorem 1. For example one may replace the second symmetric differences in (3.2) by suitable iterations of first symmetric differences, ensuring a counterpart of Lemma 1.

\section{Fractal quantities}

4.1. Fractal quantities for distributions. As said in the Introduction we wish to generalise $\lambda_{\mu}(t)$ and $s_{\mu}(t)$ according to (1.7) and (1.8), respectively, from measures with (1.6) to distributions and to ask for equalities as in the middle of (1.9). In case of measures we have (1.14) with (1.2); and hence mollifications of type (3.6) with non-negative kernels $K$ are sufficient. As a result one gets descriptions in terms of the so-called multifractal formalism (for measures). We refer to the literature mentioned in the Introduction and in [18]. If $\mu$ is replaced by arbitrary distributions $f$ then mollifications of type (3.6) with non-negative kernels are no longer sufficient and one needs wavelet transforms of type (3.7) with oscillating kernels. The extension of the multifractal formalism to (Hölder-continuous)

functions is due to S. Jaffard, [7], [8]. The wavelet transforms used there are different (such that some reproducing formulas are available). But it might be of interest that again some stabilising constructions of a similar type as in connection with the maximal function in (2.10) have been used. Our intention is different and more or less a corollary of the above considerations. First we extend $\lambda_{\mu}(t)$ and $s_{\mu}(t)$, according to (1.7) and (1.8), respectively, from measures to distributions.

Definition 2. Let $0 \leq t=\frac{1}{p}<\infty$ and

$$
f \in S^{\prime}\left(\mathbb{R}^{n}\right), \quad \operatorname{supp} f \text { compact. }
$$


(i) Let $V_{j m}^{M} f$ be given by (3.8), $\lambda \in \mathbb{R}$,

$$
V f_{p}^{\lambda, M}=\sup _{j \in \mathbb{N}_{0}} 2^{j(\lambda-n)}\left(\sum_{m \in \mathbb{Z}^{n}}\left(V_{j m}^{M} f\right)^{p}\right)^{\frac{1}{p}},
$$

and

(ii) Let

$$
\lambda^{f}(t)=\sup \left\{\lambda: V f_{p}^{\lambda, M}<\infty \text { for some } M \in \mathbb{N}_{0}\right\} .
$$

Let

$$
s_{f}(t)=\sup \left\{s: f \in B_{p \infty}^{s}\left(\mathbb{R}^{n}\right)\right\} .
$$

Remark 6. Since we assumed that $f$ has a compact support it follows that $f$ for any $p$ with $0<p \leq \infty$ belongs to some space $B_{p \infty}^{s}\left(\mathbb{R}^{n}\right)$. Furthermore, some qualitative properties of $s_{f}(t)$ are the same as for $s_{\mu}(t)$ for measures $\mu$, say, with (1.6): $s_{f}(t)$ is a concave monotonically increasing (which means not decreasing) function with respect to $t \geq 0$, Figure 1 (now not necessarily with $s_{f}(1)=0$ ). We refer for details to [18]. If for given $p$ and $M \in \mathbb{N}_{0}$,

$$
V f_{p}^{\lambda, M}<\infty \text {, then } V f_{p}^{\lambda, N}<\infty \text { for } N \geq M, N \in \mathbb{N}_{0} .
$$

This is an immediate consequence of (3.2) and (3.7), (3.8). If $M=0$ and if $f=\mu$ is a Radon measure according to (1.1) then it follows by (1.14) and (1.2) that $\lambda^{\mu}(t)=\lambda_{\mu}(t)$ according to (4.2) and (1.7) (as it should be). This explains also the factor $2^{-j n}$ in (4.1). Recall that (1.11), (1.12), is the explicit version of $\lambda_{\mu}(t)$ in (1.7). Of course, there are similar explicit expressions for $\lambda^{f}(t)$ in (4.2), where the underlying quantities in (4.1) are independent of $M$ if $M$ is large (in dependence of $f$ ).

Theorem 3. Let $0 \leq t=\frac{1}{p}<\infty$. Let $\lambda^{f}(t)$ and $s_{f}(t)$ be given by (4.2) and (4.3), respectively, where $f \in S^{\prime}\left(\mathbb{R}^{n}\right)$ has a compact support. Then

$$
s_{f}(t)=\lambda^{f}(t)+n(t-1), \quad 0 \leq t<\infty .
$$

Proof. (4.4) we may assume that $M \in \mathbb{N}_{0}$ is large. Then the above theorem is an immediate consequence of Theorem 2 and the above explanations.

4.2. Fractal quantities for measures. Let $\mu$ be a Radon measure with (1.6). Then we obtain by (1.9), (1.10) and (4.5) that

$$
\lambda_{\mu}(t)=\lambda^{\mu}(t) \text { if } 0 \leq t \leq 1 \text { and } \lambda_{\mu}(t) \leq \lambda^{\mu}(t) \text { if } t>1 .
$$

It is unclear whether one has also equaltiy in (4.6) if $t>1$.

Problem 1. For which Radon measures $\mu$ in $\mathbb{R}^{n}$ with (1.6) holds $\lambda_{\mu}(t)=\lambda^{\mu}(t)$ for all $0 \leq t<\infty$ ? 
Remark 7. One can reformulate this problem by asking of whether

$$
s_{\mu}(t)=\lambda_{\mu}(t)+n(t-1) \text { for all } 0 \leq t<\infty .
$$

We discussed this question in [18]. There are several affirmative examples with (4.7), but no counter-example. On the other hand, if $\mu$ is regular nothing like (4.7) can be expected. We refer to [18], Theorem 1.

\section{References}

[1] D.R. Adams and L.I. Hedberg, Function spaces and potential theory, Springer, Berlin, 1996.

[2] H.-Q. Bui, M. Paluszyński and M.H. Taibleson, A maximal function characterization of weighted Besov-Lipschitz and Triebel-Lizorkin spaces, Studia Math., 111 (1996), 219-246.

[3] H.-Q. Bui, M. Paluszyński and M.H. Taibleson, Characterization of Besov-Lipschitz and Triebel-Lizorkin spaces, the case $q<1$, Journ. Fourier Anal. Appl., Spec. Iss. 3 (1997), 837-846.

[4] D.E. Edmunds and H. Triebel, Function spaces, entropy numbers, differential operators, Cambridge Univ. Press, 1996.

[5] K.J. Falconer, Techniques in fractal geometry, Wiley, Chichester, 1997.

[6] Y. Heurteaux, Estimations de la dimension intérieure et de la dimension supérieure des mesures, Ann. Inst. H. Poincaré, Probab. Statist., 34 (1998), 309-338.

[7] S. Jaffard, Multifractal formalism for functions; Part I: results valid for all functions, SIAM Journ. Math. Anal., 28 (1997), 944-970.

[8] S. Jaffard, Multifractal formalism for functions; Part II: self-similar functions, SIAM Journ. Math. Anal., 28 (1997), 971-998.

[9] P. Malliavin, Integration and probability, Springer, New York, 1995.

[10] L. Olsen, A multifractal formalism, Adv. Math., 116 (1995), 82-196.

[11] J. Peetre. On spaces of Triebel-Lizorkin type, Ark. Math., 13 (1975), 123-130.

[12] T. Runst and W. Sickel, Sobolev spaces of fractional order, Nemytskij operators, and nonlinear partial differential equations, W. de Gruyter, Berlin, 1996.

[13] V.S. Rychkov, On a theorem of Bui, Paluszyński, and Taibleson, Proc. Steklov Inst. Math., 227 (1999), 280-292.

[14] H. Triebel, Theory of function spaces, Birkhäuser, Basel, 1983.

[15] H. Triebel, Theory of function spaces II, Birkhäuser, Basel, 1992.

[16] H. Triebel, Fractals and spectra, Birkhäuser, Basel, 1997. 
[17] H. Triebel, The structure of functions, Birkhäuser, Basel, 2001.

[18] H. Triebel, Fractal characteristics of measures; an approach via function spaces, Journ. Fourier Anal. Appl., 9 (2003), 411-431.

Mathematisches Institut

Fakultät für Mathematik und Informatik

Friedrich-Schiller-Universität

D-07740 Jena

Germany

(Email : triebel@minet.uni-jena.de) 


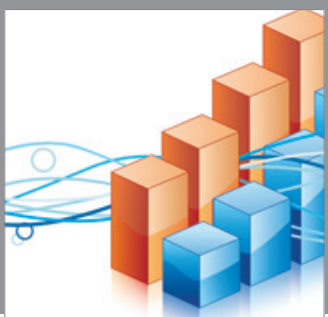

Advances in

Operations Research

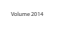

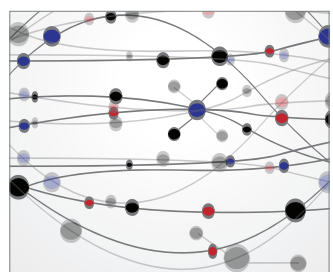

\section{The Scientific} World Journal
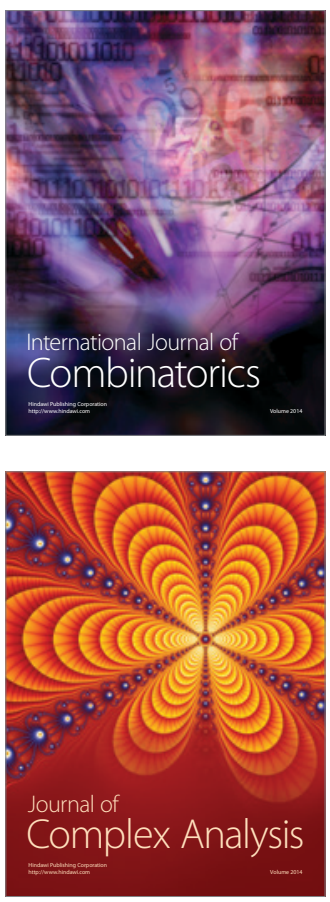

International Journal of

Mathematics and

Mathematical

Sciences
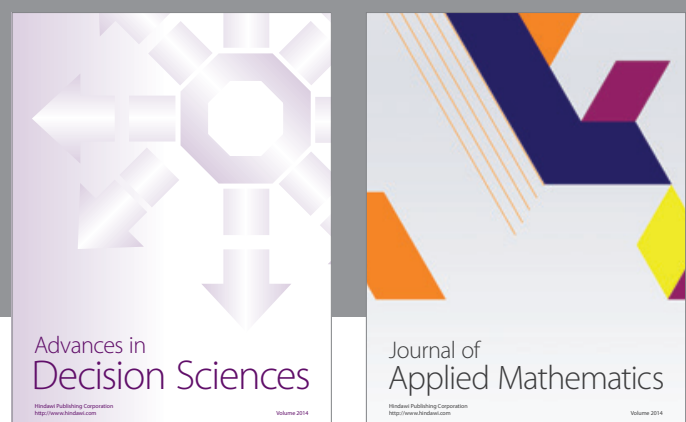

Journal of

Applied Mathematics
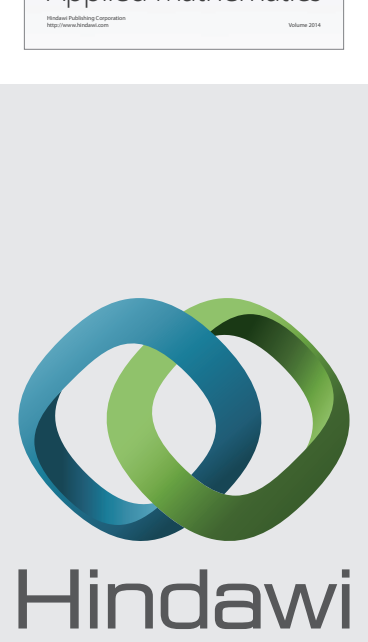

Submit your manuscripts at http://www.hindawi.com
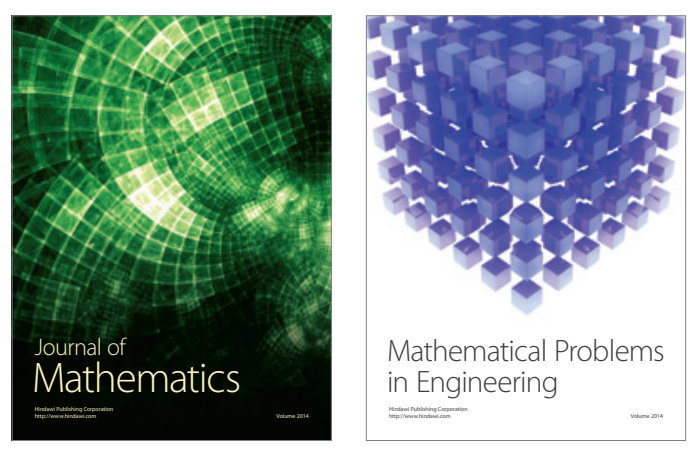

Mathematical Problems in Engineering
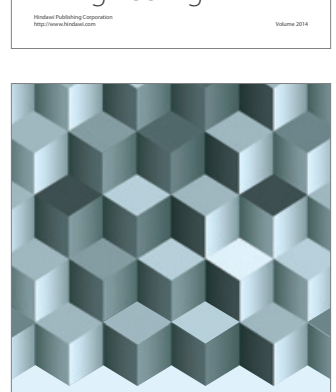

Journal of

Function Spaces
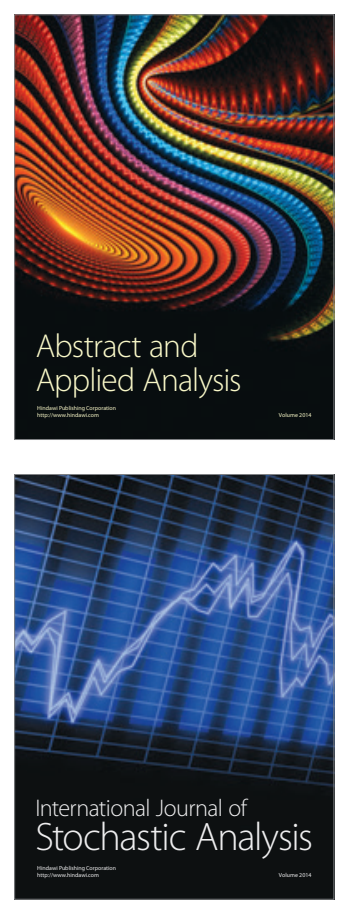

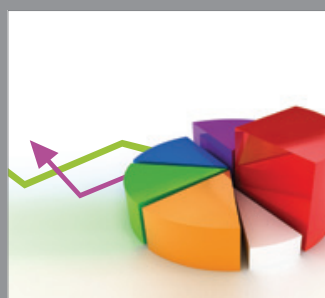

ournal of

Probability and Statistics

Promensencen
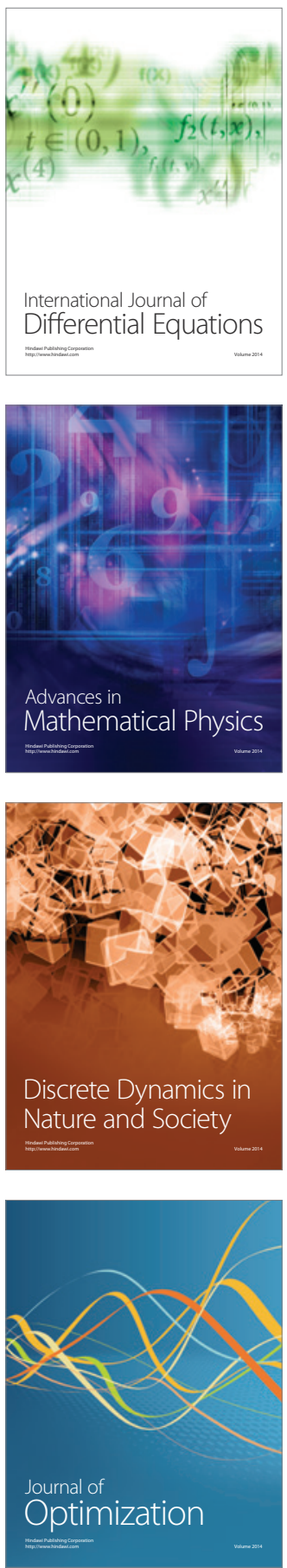